\title{
On a kinetic model for a simple market economy
}

\author{
Stephane Cordier* $\quad$ Lorenzo Pareschi ${ }^{\dagger} \quad$ Giuseppe Toscani ${ }^{\ddagger}$
}

December 21, 2004

\begin{abstract}
In this paper, we consider a simple kinetic model of economy involving both exchanges between agents and speculative trading. We show that the kinetic model admits non trivial quasi-stationary states with power law tails of Pareto type. In order to do this we consider a suitable asymptotic limit of the model yielding a Fokker-Planck equation for the distribution of wealth among individuals. For this equation the stationary state can be easily derived and shows a Pareto power law tail. Numerical results confirm the previous analysis.
\end{abstract}

Keywords. Econophysics, Boltzmann equation, wealth and income distributions, Pareto distribution.

\section{Contents}

\begin{tabular}{lll}
\hline 1 & Introduction & 2
\end{tabular}

2 A kinetic model of money asset exchanges 3

\begin{tabular}{|lll}
3 & Main properties of the kinetic equation & 4
\end{tabular}

$\begin{array}{lll}4 & \text { The continuous trading limit } & 8\end{array}$

$\begin{array}{lll}5 & \text { Numerical results } & 13\end{array}$

$\begin{array}{lll}6 & \text { Conclusions } & 14\end{array}$

* Department of Mathematics and Applications, Mathematical Physics (MAPMO)UMR 6628 University of Orléans and CNRS, 45067 Orléans, France. cordier@labomath.univ-orleans.fr

${ }^{\dagger}$ Department of Mathematics, University of Ferrara, Via Machiavelli 35 I-44100 Ferrara, Italy, and Center for Modelling Computing and Statistics, c/o Department of Economy, Institutions and Territory, University of Ferrara, Via del Gregorio 13, I-44100 Ferrara, Italy. pareschi@dm. unife.it

${ }^{\ddagger}$ Department of Mathematics, University of Pavia, via Ferrata 1, 27100 Pavia, Italy. toscani@dimat.unipv.it 


\section{Introduction}

Microscopic models of simple market economies have been recently introduced by several authors [6, 7, 11, 14]. The main idea is that an economic system composed by a sufficiently large number of agents can be described using the laws of statistical mechanics as it happens in a physical system composed of many interacting particles. The details of the economical interactions between agents characterize their wealth distribution.

The study of wealth distributions has a long history going back to more then one century ago with the Italian sociologist and economist Vilfredo Pareto which studied the distribution of income among people of different western countries and found an inverse power law 23]. More precisely if $f(w)$ is the probability density function of agents with wealth $w$ we have

$$
F(w)=\int_{w}^{\infty} f\left(w_{*}\right) d w_{*} \sim w^{-\mu} .
$$

Pareto mistakenly believed that power laws apply to the whole distribution with a universal exponent $\mu$ approximatively equal to 1.5. Later, Mandelbrot, proposed a weak Pareto law that applies only to high incomes 17 .

Income data from developed countries show Pareto-like behaviors with order 1 exponents for large values of incomes (USA $\sim 1.6$, Japan $\sim 1.8-2.2$ [11]). It is common, in fact, that $90 \%$ of the total wealth is owned by only $5 \%$ of the population. According to data, across the full range of income, we should expect the probability density function $f(w)$ to increase at low income, reach a maximum and, finally decrease with increasing wealth [11].

In our discussion we use the terms distribution of income (money) and distribution of wealth interchangeably (although the two distributions may not exactly coincide there is a strong dependence on one another). Income, in fact, is only one part of wealth, the other part being material wealth. Material products have no conservation law and their monetary value is not constant (they can be manufactured, destroyed, consumed, etc.). The distinction between the two will not influence the conclusion drawn here from the dynamical model of economic interactions we consider.

Following this line of thought, we consider here a very simple model of an open market economy involving both assets exchanges between individuals and speculative trading. In our non-stationary economy the total wealth is not conserved due to a random dynamics which describes the spontaneous growth or decrease of wealth due to investments in the stock market, housing, ect. It is important to note that this mechanism corresponds to the effects of an open market economy where the investments cause the total economy to growth (more precisely the rich would get richer and the poor would get poorer). The conservative exchanges dynamic between individuals redistributes the wealth among people.

We shall show that this kinetic model, introduced in [22], gives in a suitable asymptotic limit (hereafter called continuous trading limit) a partial differential equation of FokkerPlanck type for the distribution of money among individuals.

The equilibrium state of the Fokker-Planck equation can be computed explicitly and is of Pareto type, namely it is characterized by a power-law tail for the richest individuals. 
Our result can be related to the work [四], where a similar equation has been derived by a mean-field limit procedure.

The mathematical methods we use are close to those used in the context of kinetic theory for granular flows, where the limit procedure is known as quasi-elastic asymptotics [26]. We mention here a similar asymptotic analysis performed on a kinetic model of a simple market economy with a constant growth mechanism [25].

The paper is organized as follows. In the next section, we introduce the binary interaction between agents, which is at the basis of the kinetic model. The main properties of the model are discussed in section 3. These properties justify the continuous trading limit procedure, performed in section 4 . The limit is illustrated by several numerical results in section 5 .

\section{A kinetic model of money asset exchanges}

The goal of a kinetic model of simple market economies, is to describe the evolution of the distribution of money by means of microscopic interactions among agents or individuals which perform exchange of money. Each trade can indeed be interpreted as an interaction where a fraction of the money changes hands. We will assume that this wealth after the interaction is non negative, which corresponds to impose that no debts are allowed. This rule emphasizes the difference between economic interactions, where not all outcomes are permitted, and the classical interactions between molecules.

¿From a microscopic view point, the binary interaction is described by the following rules (we refer to [22] for more details)

$$
\begin{aligned}
w^{\prime} & =(1-\gamma) w+\gamma w_{*}+\eta w \\
w_{*}^{\prime} & =(1-\gamma) w_{*}+\gamma w+\eta_{*} w_{*}
\end{aligned}
$$

where $\left(w, w_{*}\right)$ denote the (positive) money of two arbitrary individuals before the trade and $\left(w^{\prime}, w_{*}^{\prime}\right)$ the money after the trade. In (1) we will not allow agents to have debts, and thus the interaction takes place only if $w^{\prime} \geq 0$ and $w_{*}^{\prime} \geq 0$. In (11) the transaction coefficient $\gamma \in(0,1)$ is a given constant, while $\eta$ and $\eta_{*}$ are random variables with the same distribution (for example normal) with variance $\sigma^{2}$ and zero mean.

Let us describe the three terms in the right hand side. The first term is related to the marginal saving propensity of the agents, the second corresponds to the money transaction, and the last contains the effects of an open economy describing the market returns. Note that since debts are not allowed, the total amount of money in the system is increasing.

This binary interaction model can be also related to some recent Lotka-Volterra type models 沺. A similar trade rule where $\eta$ and $\eta_{*}$ have the same positive constant value has been considered recently in [25]. In a closed economical system it is assumed that the total amount of money is conserved $\left(\eta, \eta_{*} \equiv 0\right)$. This conservation law is reminiscent of analogous conservations which take place in kinetic theory. In such a situation equations (1) correspond to a granular gas like model (or to a traffic flow model) where the stationary state is a Dirac delta centered in the average wealth (usually referred to as synchronized 
traffic state in traffic flow modelling). Thus all agents will end up in the market with exactly the same amount of money.

Let $f(w, t)$ denote the distribution of money $w \in \mathbb{R}_{+}$at time $t \geq 0$. By standard methods of kinetic theory, it is easy to show that the time evolution of $f$ is driven by the following integro-differential equation of Boltzmann type,

$$
\frac{\partial f}{\partial t}=\int_{\mathbb{R}^{2}} \int_{0}^{\infty}\left(\beta_{\left({ }^{\prime} w,{ }^{\prime} w_{*}\right) \rightarrow\left(w, w_{*}\right)} J f\left({ }^{\prime} w\right) f\left({ }^{\prime} w_{*}\right)-\beta_{\left(w, w_{*}\right) \rightarrow\left(w^{\prime}, w_{*}^{\prime}\right)} f(w) f\left(w_{*}\right) d w_{*} d \eta d \eta_{*}\right.
$$

where $\left(' w, w_{*}\right)$ are the pre-trading money that generates the couple $\left(w, w_{*}\right)$ after the interaction. In (2) $J$ is jacobian of the transformation of $\left(w, w_{*}\right)$ into $\left(w^{\prime}, w_{*}^{\prime}\right)$ and the kernel $\beta$ is related to the details of the binary interaction.

We shall restrict here to a transition rate of the form

$$
\beta_{\left(w, w_{*}\right) \rightarrow\left(w^{\prime}, w_{*}^{\prime}\right)}=\mu(\eta) \mu\left(\eta_{*}\right) \Psi\left(w^{\prime} \geq 0\right) \Psi\left(w_{*}^{\prime} \geq 0\right),
$$

where $\Psi(A)$ is the indicator function of the set $A$, and $\mu(\cdot)$ is a symmetric probability density with zero mean and variance $\sigma^{2}$. The rate function $\beta_{\left(w^{\prime}, w_{*}^{\prime}\right) \rightarrow\left(w, w_{*}\right)}$ characterizes the effects of the open economy through the distribution of the random variables $\eta$ and $\eta_{*}$ and takes into account the hypothesis that no-debts are allowed. The above equation can be included in a more general settings where the trade rule has a more complex structure including, for example, risk, taxes and subsidies as described in [22].

We remark that, for general probability density $\mu(\cdot)$, the rate function $\beta$ depends on the wealth variables $\left(w, w_{*}\right)$ through the indicator functions $\Psi$. This is analogous to what happens in the classical Boltzmann equation [5], where the rate function depends on the relative velocity. A simplified situation occurs when the random variables take values on the set $(-(1-\gamma), 1-\gamma)$. In this case in fact, both $w^{\prime} \geq 0$ and $w_{*}^{\prime} \geq 0$, and the kernel $\beta$ does not depend on the wealth variables $\left(w, w_{*}\right)$. In this case the kinetic equation (2) is the corresponding of the classical Boltzmann equation for Maxwell molecules [2], which presents several mathematical simplifications. In all cases however, methods borrowed from kinetic theory of rarefied gas can be used to study the evolution of the function $f$.

\section{Main properties of the kinetic equation}

We will start our analysis by introducing some notations and by discussing the main properties of the kinetic equation (2). Let $Q(f, f)$ denote the interaction integral,

$Q(f, f)(w)=\int_{\mathbb{R}^{2}} \int_{0}^{\infty}\left(\beta_{\left({ }^{\prime} w,{ }^{\prime} w_{*}\right) \rightarrow\left(w, w_{*}\right)} J f\left({ }^{\prime} w\right) f\left({ }^{\prime} w_{*}\right)-\beta_{\left(w, w_{*}\right) \rightarrow\left(w^{\prime}, w_{*}^{\prime}\right)} f(w) f\left(w_{*}\right) d w_{*} d \eta d \eta_{*}\right.$.

Let $\mathcal{M}_{0}$ the space of all probability measures in $\mathbb{R}_{+}$and by

$$
\mathcal{M}_{p}=\left\{\mu \in \mathcal{M}_{0}: \int_{\mathbb{R}_{+}}|v|^{p} \mu(d v)<+\infty, p \geq 0\right\},
$$

the space of all Borel probability measures of finite momentum of order $p$, equipped with the topology of the weak convergence of the measures. 
Let $\mathcal{F}_{s}\left(\mathbb{R}_{+}\right)$, be the class of all real functions on $\mathbb{R}_{+}$such that $g(0)=g^{\prime}(0)=0$, and $g^{(m)}(v)$ is Hölder continuous of order $\delta$,

$$
\left\|g^{(m)}\right\|_{\delta}=\sup _{v \neq w} \frac{\left|g^{(m)}(v)-g^{(m)}(w)\right|}{|v-w|^{\delta}}<\infty
$$

the integer $m$ and the number $0<\delta \leq 1$ are such that $m+\delta=s$, and $g^{(m)}$ denotes the $m$-th derivative of $g$.

In the rest of the paper we will assume that the symmetric probability density $\mu(\eta)$ which characterizes the transition rate belongs to $\mathcal{M}_{2+\alpha}$, for some $\alpha>0$. Moreover, to simplify the computations, we assume that this density is obtained from a given random variable $Y$ with zero mean and unit variance, that belongs to $\mathcal{M}_{2+\alpha}$. Thus, $\mu$ of variance $\sigma^{2}$ is the density of $\sigma Y$. By this assumption, we can easily obtain the dependence on $\sigma$ of the moments of $\mu$. In fact, for any $p>2$ such that the $p$-th moment of $Y$ exists,

$$
\int_{\mathbb{R}}|\eta|^{p} \mu(\eta) d \eta=E\left(|\sigma Y|^{p}\right)=\sigma^{p} E\left(|Y|^{p}\right) .
$$

By a weak solution of the initial value problem for equation (2), corresponding to the initial probability density $f_{0}(w) \in \mathcal{M}_{p}, p>1$ we shall mean any probability density $f \in C^{1}\left(\mathbb{R}_{+}, \mathcal{M}_{p}\right)$ satisfying the weak form of the equation

$$
\begin{aligned}
& \frac{d}{d t} \int_{\mathbb{R}_{+}} \phi(w) f(w, t) d w=(Q(f, f), \phi)= \\
& \int_{\mathbb{R}^{2}} \int_{\mathbb{R}_{+}^{2}} \beta_{\left(w, w_{*}\right) \rightarrow\left(w^{\prime}, w_{*}^{\prime}\right)} f(w) f\left(w_{*}\right)\left(\phi\left(w^{\prime}\right)-\phi(w)\right) d w_{*} d w d \eta d \eta_{*}
\end{aligned}
$$

for $t>0$ and all $\phi \in \mathcal{F}_{p}\left(\mathbb{R}_{+}\right)$, and such that for all $\phi \in \mathcal{F}_{p}\left(\mathbb{R}_{+}\right)$

$$
\lim _{t \rightarrow 0} \int_{\mathbb{R}_{+}} \phi(w) f(w, t) d w=\int_{\mathbb{R}_{+}} \phi(w) f_{0}(w) d w .
$$

We can alternatively use the symmetric form

$$
\begin{aligned}
\frac{d}{d t} \int_{0}^{\infty} f(w) \phi(w) d w= & \frac{1}{2} \int_{\mathbb{R}^{2}} \int_{\mathbb{R}_{+}^{2}} \beta_{\left(w, w_{*}\right) \rightarrow\left(w^{\prime}, w_{*}^{\prime}\right)} f(w) f\left(w_{*}\right) \\
& \left(\phi\left(w^{\prime}\right)+\phi\left(w_{*}^{\prime}\right)-\phi(w)-\phi\left(w_{*}\right)\right) d w_{*} d w d \eta d \eta_{*} .
\end{aligned}
$$

Existence of a weak solution to the initial value problem for equation (2) can be easily obtained by using methods first applied to the Boltzmann equation [5]. The main difference here is that the classical Boltzmann $H$-theorem, which prevents formation of concentration, does not hold.

¿From (6) (or equivalently from (8) ) conservation of the total number of agents is obtained for $\phi(w)=1$ which represents the only conservation property satisfied by the 
system. The choice $\phi(w)=w$ is of particular interest since it gives the time evolution of the total wealth. We have

$$
\begin{gathered}
\frac{d}{d t} \int_{\mathbb{R}_{+}} w f(w, t) d w=\int_{\mathbb{R}^{2}} \int_{\mathbb{R}_{+}^{2}} \beta_{\left(w, w_{*}\right) \rightarrow\left(w^{\prime}, w_{*}^{\prime}\right)} f(w) f\left(w_{*}\right)\left(\gamma\left(w_{*}-w\right)+\eta w\right) d w_{*} d w d \eta d \eta_{*}= \\
\quad-\int_{\mathbb{R}^{2}} \int_{\mathbb{R}_{+}^{2}} \eta \mu(\eta) \mu\left(\eta_{*}\right)\left(1-\Psi\left(w^{\prime} \geq 0\right) \Psi\left(w_{*}^{\prime} \geq 0\right)\right) w f(w) f\left(w_{*}\right) d w_{*} d w d \eta d \eta_{*} .
\end{gathered}
$$

Since $\eta<0$ on the set where $\Psi\left(w^{\prime} \geq 0\right)=0$, equation (9) shows that the total wealth is increasing, unless $w^{\prime} \geq 0$ and $w_{*}^{\prime} \geq 0$ for all exchanges (in such case the total wealth would be conserved). An upper bound for the total growth can be derived considering that $1-\gamma+\eta \geq 0$ implies $w^{\prime} \geq 0$ (respectively $1-\gamma+\eta_{*} \geq 0$ implies $w_{*}^{\prime} \geq 0$ ). Thus,

$$
1-\Psi\left(w^{\prime} \geq 0\right) \Psi\left(w_{*}^{\prime} \geq 0\right) \leq \Psi(1-\gamma+\eta<0)+\Psi\left(1-\gamma+\eta_{*}<0\right) .
$$

Substituting this inequality into (9) we obtain

$$
\begin{gathered}
\frac{d}{d t} \int_{\mathbb{R}_{+}} w f(w, t) d w \leq-\int_{\mathbb{R}^{2}} \eta \mu(\eta) \mu\left(\eta_{*}\right)(\Psi(1-\gamma+\eta<0) \\
\left.+\Psi\left(1-\gamma+\eta_{*}<0\right)\right) d \eta d \eta_{*} \int_{\mathbb{R}_{+}} w f(w) d w .
\end{gathered}
$$

By Markov inequality,

$$
\int_{\mathbb{R}} \mu\left(\eta_{*}\right) d \eta_{*} \Psi\left(1-\gamma+\eta_{*}<0\right) \leq \frac{\sigma^{2+\alpha}}{(1-\gamma)^{2+\alpha}}
$$

while

$$
\int_{\mathbb{R}}|\eta| \mu(\eta) d \eta \Psi(1-\gamma+\eta<0) \leq \frac{1}{(1-\gamma)^{1+\alpha}} \int_{\mathbb{R}}|\eta|^{2+\alpha} \mu(\eta) d \eta \Psi(1-\gamma+\eta<0) \leq \frac{\sigma^{2+\alpha}}{(1-\gamma)^{1+\alpha}} .
$$

Finally,

$$
\frac{d}{d t} \int_{\mathbb{R}_{+}} w f(w, t) d w \leq \frac{\sigma^{2+\alpha}(2-\gamma)}{(1-\gamma)^{2+\alpha}} \int_{\mathbb{R}_{+}} w f(w, t) d w .
$$

We remark that, if the kernel $\beta$ does not depend on the wealth variables, the kinetic model preserves the total wealth.

Similar bounds for moments of order higher than 2 can be obtained in a similar way. To this purpose, let us fix $\phi(w)=w^{p}$ for some $p>2$. Using the same trick as in (9) we obtain

$$
\begin{gathered}
\frac{d}{d t} \int_{\mathbb{R}_{+}} w^{p} f(w, t) d w=\int_{\mathbb{R}^{2}} \int_{\mathbb{R}_{+}^{2}} \beta_{\left(w, w_{*}\right) \rightarrow\left(w^{\prime}, w_{*}^{\prime}\right)} f(w) f\left(w_{*}\right)\left(\left|w^{\prime}\right|^{p}-w^{p}\right) d w_{*} d w d \eta d \eta_{*}= \\
\int_{\mathbb{R}^{2}} \int_{\mathbb{R}_{+}^{2}} \mu(\eta) \mu\left(\eta_{*}\right) f(w) f\left(w_{*}\right)\left(\left|w^{\prime}\right|^{p}-w^{p}\right) d w_{*} d w d \eta d \eta_{*}+
\end{gathered}
$$




$$
\begin{gathered}
-\int_{\mathbb{R}^{2}} \int_{\mathbb{R}_{+}^{2}} \mu(\eta) \mu\left(\eta_{*}\right)\left(1-\Psi\left(w^{\prime} \geq 0\right) \Psi\left(w_{*}^{\prime} \geq 0\right)\right)\left(\left|w^{\prime}\right|^{p}-w^{p}\right) f(w) f\left(w_{*}\right) d w_{*} d w d \eta d \eta_{*} \leq \\
\int_{\mathbb{R}^{2}} \int_{\mathbb{R}_{+}^{2}} \mu(\eta) \mu\left(\eta_{*}\right) f(w) f\left(w_{*}\right)\left(\left|w^{\prime}\right|^{p}-w^{p}\right) d w_{*} d w d \eta d \eta_{*}+ \\
+\int_{\mathbb{R}^{2}} \int_{\mathbb{R}_{+}^{2}} \mu(\eta) \mu\left(\eta_{*}\right)\left(1-\Psi\left(w^{\prime} \geq 0\right) \Psi\left(w_{*}^{\prime} \geq 0\right)\right) w^{p} f(w) f\left(w_{*}\right) d w_{*} d w d \eta d \eta_{*} .
\end{gathered}
$$

Using again Markov inequality we obtain

$$
\begin{gathered}
\int_{\mathbb{R}^{2}} \int_{\mathbb{R}_{+}^{2}} \mu(\eta) \mu\left(\eta_{*}\right)\left(1-\Psi\left(w^{\prime} \geq 0\right) \Psi\left(w_{*}^{\prime} \geq 0\right)\right) w^{p} f(w) f\left(w_{*}\right) d w_{*} d w d \eta d \eta_{*} \leq \\
\int_{\mathbb{R}^{2}} \mu(\eta) \mu\left(\eta_{*}\right)\left(\Psi(1-\gamma+\eta<0)+\Psi\left(1-\gamma+\eta_{*}<0\right)\right) d \eta d \eta_{*} \int_{\mathbb{R}_{+}} w^{p} f(w) d w \leq \\
2 \frac{\sigma^{2+\alpha}}{(1-\gamma)^{2+\alpha}} \int_{\mathbb{R}_{+}} w^{p} f(w) d w .
\end{gathered}
$$

Moreover, we can write

$$
\left|w^{\prime}\right|^{p}=w^{p}+p w^{p-1}\left(w^{\prime}-w\right)+\frac{1}{2} p(p-1)|\tilde{w}|^{p-2}\left(w^{\prime}-w\right)^{2},
$$

where, for some $0 \leq \theta \leq 1$

$$
\tilde{w}=\theta w^{\prime}+(1-\theta) w .
$$

Hence,

$$
\begin{gathered}
\int_{\mathbb{R}^{2}} \int_{\mathbb{R}_{+}^{2}} \mu(\eta) \mu\left(\eta_{*}\right) f(w) f\left(w_{*}\right)\left(\left|w^{\prime}\right|^{p}-w^{p}\right) d w_{*} d w d \eta d \eta_{*}= \\
\int_{\mathbb{R}^{2}} \int_{\mathbb{R}_{+}^{2}} \mu(\eta) \mu\left(\eta_{*}\right) f(w) f\left(w_{*}\right)\left(p w^{p-1}\left(w^{\prime}-w\right)+\frac{1}{2} p(p-1)|\tilde{w}|^{p-2}\left(w^{\prime}-w\right)^{2}\right) d w_{*} d w d \eta d \eta_{*} \leq \\
\frac{1}{2} p(p-1) \int_{\mathbb{R}^{2}} \int_{\mathbb{R}_{+}^{2}} \mu(\eta) \mu\left(\eta_{*}\right) f(w) f\left(w_{*}\right)|\tilde{w}|^{p-2}\left(w^{\prime}-w\right)^{2} d w_{*} d w d \eta d \eta_{*} .
\end{gathered}
$$

In fact,

$$
\begin{gathered}
\int_{\mathbb{R}^{2}} \int_{\mathbb{R}_{+}^{2}} \mu(\eta) \mu\left(\eta_{*}\right) f(w) f\left(w_{*}\right) w^{p-1}\left(w^{\prime}-w\right) d w_{*} d w d \eta d \eta_{*}= \\
\int_{\mathbb{R}^{2}} \int_{\mathbb{R}_{+}^{2}} \mu(\eta) \mu\left(\eta_{*}\right) f(w) f\left(w_{*}\right) w^{p-1}\left(\gamma\left(w_{*}-w\right)+\eta w\right) d w_{*} d w d \eta d \eta_{*}= \\
\gamma \int_{\mathbb{R}_{+}^{2}} f(w) f\left(w_{*}\right) w^{p-1}\left(w_{*}-w\right) d w_{*} d w \leq 0 .
\end{gathered}
$$


The last bound follows by Hölder inequality. In fact,

$$
\begin{gathered}
\int_{\mathbb{R}_{+}^{2}} f(w) f\left(w_{*}\right) w^{p-1} w_{*} d w_{*} d w=\int_{\mathbb{R}_{+}} f(w) w^{p-1} d w \int_{\mathbb{R}_{+}} f\left(w_{*}\right) w_{*} d w_{*} \leq \\
\left(\int_{\mathbb{R}_{+}} f(w) w^{p} d w\right)^{(p-1) / p}\left(\int_{\mathbb{R}_{+}} f\left(w_{*}\right) w_{*}^{p} d w_{*}\right)^{1 / p}=\int_{\mathbb{R}_{+}} f(w) w^{p} d w .
\end{gathered}
$$

Finally, since

$$
|\tilde{w}|^{p-2}=\left|(1-\theta \gamma) w+\theta \gamma w_{*}+\theta \eta w\right|^{p-2} \leq c_{p}\left[w^{p-2}+\gamma^{p-2} w_{*}^{p-2}+|\eta|^{p-2} w^{p-2}\right],
$$

we obtain

$$
\begin{gathered}
\int_{\mathbb{R}^{2}} \int_{\mathbb{R}_{+}^{2}} \mu(\eta) \mu\left(\eta_{*}\right) f(w) f\left(w_{*}\right)|\tilde{w}|^{p-2}\left(w^{\prime}-w\right)^{2} d w_{*} d w d \eta d \eta_{*} \leq \\
c_{p} \int_{\mathbb{R}^{2}} \int_{\mathbb{R}_{+}^{2}} \mu(\eta) \mu\left(\eta_{*}\right) f(w) f\left(w_{*}\right)\left[w^{p-2}+\gamma^{p-2} w_{*}^{p-2}+|\eta|^{p-2} w^{p-2}\right] \cdot \\
\cdot\left[\gamma^{2}\left(w_{*}-w\right)^{2}+\eta^{2} w^{2}+2 \eta w\left(w_{*}-w\right)\right] d w_{*} d w d \eta d \eta_{*} \leq \\
c_{p} A_{p}(\sigma, \gamma) \int_{\mathbb{R}_{+}} w^{p} f(w) d w
\end{gathered}
$$

where

$$
A_{p}(\sigma, \gamma)=\sigma^{2}\left(1+\gamma^{p-2}\right)+\sigma^{p}+2 \gamma^{2}+2 \gamma^{p}+2 \gamma^{2} \sigma^{p-2} .
$$

Grouping all estimates together, we finally obtain the bound for the moments

$$
\frac{d}{d t} \int_{\mathbb{R}_{+}} w^{p} f(w, t) d w \leq\left(\frac{1}{2} p(p-1) c_{p} A_{p}(\sigma, \gamma)+2 \frac{\sigma^{2+\alpha}}{(1-\gamma)^{2+\alpha}}\right) \int_{\mathbb{R}_{+}} w^{p} f(w) d w .
$$

\section{The continuous trading limit}

The previous analysis shows that in general it is quite difficult both to study in details the evolution of the wealth function, and to describe the asymptotic behavior. As is usual in kinetic theory, particular asymptotics of the equation result in simplified models (generally of Fokker-Planck type), for which it is relatively easier to find steady states, and to prove their stability. In order to give a physical basis on these asymptotics, it is relevant to discuss in some detail the interaction rule (11). To skip inessential difficulties, that as we will see later on do not change the structure of the limit equation, we suppose here that the random variables take values on the set $(-(1-\gamma), 1-\gamma)$. As remarked in the previous section, in this case both $w^{\prime} \geq 0$ and $w_{*}^{\prime} \geq 0$, and the kernel $\beta$ does not depend on the wealth variables $\left(w, w_{*}\right)$. Let us denote by $E(X)$ the mathematical expectation of the random variable $X$. Then the following properties follow from (11)

$$
E\left[w^{\prime}+w_{*}^{\prime}\right]=w+w_{*}, \quad E\left[w^{\prime}-w_{*}^{\prime}\right]=(1-2 \gamma)\left(w-w_{*}\right) .
$$


The first equality in (20) describes the property of mean conservation of wealth. The second refers to the tendency of the trade to decrease (in mean) the distance between wealths after the interaction. This tendency is a universal consequence of the rule (1), in that it holds whatever distribution one assigns to $\mu$, namely to the random variable which accounts for the effects of the market returns in an open economy. This universality is false for the first equality, which in general has to be substituted by the inequality

$$
E\left[w^{\prime}+w_{*}^{\prime}\right] \geq w+w_{*} .
$$

Hence, in general the effects of the market returns in an open economy account for an increasing of the microscopic mean wealth.

The second property in $(20)$ is analogous to the similar one that holds in a collision between molecules in a granular gas. There the quantity $e=2 \gamma$ is called "coefficient of restitution", and describes the peculiar fact that energy is dissipated [26]. If we want to consider the situation in which most of the trades corresponds to a very small exchange of money $(\gamma \rightarrow 0)$, and at the same time we want to maintain both properties $(20)$ at a macroscopic level, we have to pretend that

$$
\int_{\mathbb{R}_{+}^{2}}\left(w+w_{*}\right) f(w) f\left(w_{*}\right) d w d w_{*}=2 \int_{\mathbb{R}_{+}} w f(w) d w=2 m(t)
$$

remains constant, while

$$
\int_{\mathbb{R}_{+}^{2}}\left(w-w_{*}\right)^{2} f(w) f\left(w_{*}\right) d w d w_{*}=A_{f}(t)
$$

is varying with time, and decays to zero when the market returns are not present (i.e. $\sigma=0)$.

When the kernel $\beta$ does not depend on the wealth variables, (9) implies that $m(t)=m_{0}$. Moreover, explicit computations show that $A_{f}(t)$ varies with law

$$
\frac{d A_{f}(t)}{d t}=-4\left[\gamma(1-\gamma)-\frac{\sigma^{2}}{2}\right] A_{f}(t)+2 \sigma^{2} m^{2}
$$

Hence, when $\sigma=0, A_{f}(t)$ decays exponentially to zero. It is now evident that (22) is satisfied for any value of $\gamma$ and $\sigma$, while (23) looses its meaning as $\gamma$ an $\sigma$ tend to zero. Of course, one can rewrite (24) as

$$
\frac{d A_{f}(t)}{d t}=-4 \gamma\left[\left(1-\gamma-\frac{\sigma^{2}}{2 \gamma}\right) A_{f}(t)-\frac{\sigma^{2}}{2 \gamma} m^{2}\right] .
$$

Hence, if we set

$$
\tau=\gamma t, \quad g(w, \tau)=f(w, t)
$$

which implies $f_{0}(w)=g_{0}(w)$, we obtain

$$
\frac{d A_{g}(\tau)}{d \tau}=-4\left(1-\gamma-\frac{\sigma^{2}}{2 \gamma}\right) A_{g}(\tau)+2 \frac{\sigma^{2}}{\gamma} m^{2} .
$$


Letting now both $\gamma \rightarrow 0$ and $\sigma \rightarrow 0$ in such a way that $\sigma^{2} / \gamma=\lambda$, (27) becomes in the limit

$$
\frac{d A_{g}(\tau)}{d \tau}=-(4-2 \lambda) A_{g}(\tau)+2 \lambda m^{2}
$$

This formal argument shows that the value of the ratio $\sigma^{2} / \gamma$ is of paramount importance to get asymptotics which maintain memory of the microscopic interactions. In particular, while for $\lambda<2 A_{g}(\tau)$ converges to the finite value $\bar{A}_{g}=\lambda m^{2} /(2-\lambda), A_{g}(\tau)$ diverges to infinity as time goes to infinity when $\lambda \geq 2$.

In the remaining of this section, we shall present a rigorous derivation of a FokkerPlanck model from the Boltzmann equation for the wealth function $g(w, \tau)$, when both $\gamma \rightarrow 0$ and $\sigma \rightarrow 0$ in such a way that $\sigma^{2} / \gamma \rightarrow \lambda$. This derivation, which is similar to the quasi-elastic limit of granular gases, is of major relevance for the study of the asymptotic equilibrium states of the kinetic model. First, we show how the Fokker-Planck equation comes out for the simpler case of a kernel which does not depend on the wealth variables. Second, we extend the result to a general kernel.

The scaled density $g(v, \tau)=f(v, t)$ satisfies the weak form

$$
\frac{d}{d \tau} \int_{0}^{\infty} g \phi d w=\frac{1}{\gamma} \int_{\mathbb{R}^{2}} \int_{\mathbb{R}_{+}^{2}} \mu(\eta) \mu\left(\eta_{*}\right) g(w) g\left(w_{*}\right)\left(\phi\left(w^{\prime}\right)-\phi(w)\right) d w_{*} d w d \eta d \eta_{*} .
$$

Given $0<\delta \leq \alpha$, let us set $\phi \in \mathcal{F}_{2+\delta}\left(\mathbb{R}_{+}\right)$.

By (1),

$$
w^{\prime}-w=\gamma\left(w_{*}-w\right)+\eta w .
$$

Then, if we use a second order Taylor expansion of $\phi$ around $w$

$$
\phi\left(w^{\prime}\right)-\phi(w)=\left(\gamma\left(w_{*}-w\right)+\eta w\right) \phi^{\prime}(w)+\frac{1}{2}\left(\gamma\left(w_{*}-w\right)+\eta w\right)^{2} \phi^{\prime \prime}(\tilde{w}),
$$

where, for some $0 \leq \theta \leq 1$

$$
\tilde{w}=\theta w^{\prime}+(1-\theta) w .
$$

Inserting this expansion in the collision operator, we get

$$
\begin{aligned}
& \frac{d}{d \tau} \int_{0}^{\infty} g \phi d w=\frac{1}{\gamma} \int_{\mathbb{R}^{2}} \int_{\mathbb{R}_{+}^{2}} \mu(\eta) \mu\left(\eta_{*}\right)\left[\left(\gamma\left(w_{*}-w\right)+\eta w\right) \phi^{\prime}(w)+\right. \\
& \left.+\frac{1}{2}\left(\gamma\left(w_{*}-w\right)+\eta w\right)^{2} \phi^{\prime \prime}(w)\right] g\left(w_{*}\right) g(w) d w_{*} d w d \eta d \eta_{*}+R(\gamma, \sigma),
\end{aligned}
$$

where

$$
\begin{aligned}
R(\gamma, \sigma)= & \frac{1}{2 \gamma} \int_{\mathbb{R}^{2}} \int_{\mathbb{R}_{+}^{2}} \mu(\eta) \mu\left(\eta_{*}\right)\left(\gamma\left(w_{*}-w\right)+\eta w\right)^{2} . \\
& \cdot\left(\phi^{\prime \prime}(\tilde{w})-\phi^{\prime \prime}(w)\right) g\left(w_{*}\right) g(w) d w_{*} d w d \eta d \eta_{*}
\end{aligned}
$$

Since $\phi \in \mathcal{F}_{2+\delta}\left(\mathbb{R}_{+}\right)$, and $|\tilde{w}-w|=\theta\left|w^{\prime}-w\right|$

$$
\left|\phi^{\prime \prime}(\tilde{w})-\phi^{\prime \prime}(w)\right| \leq\left\|\phi^{\prime \prime}\right\|_{\delta}|\tilde{w}-w|^{\delta} \leq\left\|\phi^{\prime \prime}\right\|_{\delta}\left|w^{\prime}-w\right|^{\delta} .
$$


Hence

$$
\begin{aligned}
|R(\gamma, \sigma)| \leq & \frac{\left\|\phi^{\prime \prime}\right\|_{\delta}}{2 \gamma} \int_{\mathbb{R}^{2}} \int_{\mathbb{R}_{+}^{2}} \mu(\eta) \mu\left(\eta_{*}\right) . \\
& \cdot\left|\gamma\left(w_{*}-w\right)+\eta w\right|^{2+\delta} g\left(w_{*}\right) g(w) d w_{*} d w d \eta d \eta_{*}
\end{aligned}
$$

By virtue of the inequality

$$
\left|\gamma\left(w_{*}-w\right)+\eta w\right|^{2+\delta} \leq 4^{1+\delta}\left(\left|\gamma w_{*}\right|^{2+\delta}+|\gamma w|^{2+\delta}+|\eta w|^{2+\delta}\right)
$$

we finally obtain the bound

$$
|R(\gamma, \sigma)| \leq 2^{1+2 \delta}\left\|\phi^{\prime \prime}\right\|_{\delta}\left(2 \gamma^{1+\delta}+\frac{1}{\gamma} \int_{\mathbb{R}}|\eta|^{2+\delta} \mu(\eta) d \eta\right) \int_{\mathbb{R}_{+}} w^{2+\delta} g(w) d w
$$

Since $\mu$ is a probability density with zero mean and $\lambda \gamma$ variance, and $\mu$ belongs to $\mathcal{M}_{2+\alpha}$, for $\alpha>\delta$,

$$
\int_{\mathbb{R}}|\eta|^{2+\delta} \mu(\eta) d \eta=E\left(|\sqrt{\lambda \gamma} Y|^{2+\delta}\right)=(\lambda \gamma)^{1+\delta / 2} E\left(|Y|^{2+\delta}\right)
$$

and $E\left(|Y|^{2+\delta}\right)$ is bounded. Using this equality into (32) one shows that $R(\gamma, \sigma)$ converges to zero as $\gamma \rightarrow 0$, if $\int_{\mathbb{R}_{+}} w^{2+\delta} g(w, \tau)$ remains bounded at any fixed time $\tau>0$, provided the same bound holds at time $\tau=0$. By virtue of bound (19), taking $\sigma^{2}=\lambda \gamma$, we obtain

$$
\begin{gathered}
\frac{d}{d \tau} \int_{\mathbb{R}_{+}} w^{2+\delta} g(w, \tau) d w \leq \\
\frac{1}{\gamma}\left(\frac{1}{2} p(p-1) c_{2+\delta} A_{2+\delta}(\sqrt{\lambda \gamma}, \gamma)+2 \frac{(\lambda \gamma)^{1+\alpha / 2}}{(1-\gamma)^{2+\alpha}}\right) \int_{\mathbb{R}_{+}} w^{p} g(w, \tau) d w .
\end{gathered}
$$

Since the lower order term in $A_{2+\delta}(\sqrt{\lambda \gamma}, \gamma)$ is $\lambda \gamma(1-\gamma)^{\delta}$, it follows that the boundedness of the moment holds independently of the value of $\gamma$. Therefore, at any fixed time $\tau$

$$
|R(\gamma, \sigma)|(\tau) \leq 2^{1+2 \delta}\left\|\phi^{\prime \prime}\right\|_{\delta}\left(2 \gamma^{1+\delta}+\lambda^{2+\delta} \gamma^{\delta / 2} E\left(|Y|^{2+\delta}\right)\right) C \tau \int_{\mathbb{R}_{+}} w^{2+\delta} g_{0}(w) . d w
$$

Hence, the remainder $R(\gamma, \sigma)$ converges to zero as both $\gamma$ and $\sigma$ converge to zero, in such a way that $\sigma^{2}=\lambda \gamma$. Within the same scaling,

$$
\begin{gathered}
\lim _{\gamma \rightarrow 0} \frac{1}{\gamma} \int_{\mathbb{R}^{2}} \int_{\mathbb{R}_{+}^{2}} \mu(\eta) \mu\left(\eta_{*}\right)\left[\left(\gamma\left(w_{*}-w\right)+\eta w\right) \phi^{\prime}(w)+\right. \\
\left.+\frac{1}{2}\left(\gamma\left(w_{*}-w\right)+\eta w\right)^{2} \phi^{\prime \prime}(w)\right] g\left(w_{*}\right) g(w) d w_{*} d w d \eta d \eta_{*}= \\
\int_{\mathbb{R}_{+}}\left[(m-w) \phi^{\prime}(w)+\frac{\lambda}{2} w^{2} \phi^{\prime \prime}(w)\right] g(w) d w
\end{gathered}
$$


The right-hand side of (35) is nothing but the weak form of the Fokker-Planck equation

$$
\frac{\partial g}{\partial \tau}=\frac{\lambda}{2} \frac{\partial^{2}}{\partial w^{2}}\left(w^{2} g\right)+\frac{\partial}{\partial w}((w-m) g)
$$

The limit Fokker-Planck equation can be rewritten as

$$
\left.\frac{\partial g}{\partial \tau}=\frac{\partial}{\partial w}\left[(w-m)+\frac{\lambda}{2} w\right) g+\frac{\lambda}{2} w \frac{\partial}{\partial w}(w g)\right] .
$$

The general case of a wealth-variables depending rate function can be easily obtained from the previous computations. With respect to formula (30) we have to consider two more terms in the remainder. The first one comes out from the possibility to have negative wealth variables as outcome of the interaction. This term reads

$$
\begin{gathered}
R_{1}(\gamma, \sigma)=-\frac{1}{\gamma} \int_{\mathbb{R}^{2}} \int_{\mathbb{R}_{+}^{2}} \mu(\eta) \mu\left(\eta_{*}\right) . \\
\cdot\left(1-\Psi\left(w^{\prime} \geq 0\right) \Psi\left(w_{*}^{\prime} \geq 0\right)\right)\left(\phi\left(w^{\prime}\right)-\phi(w)\right) g(w) g\left(w_{*}\right) d w_{*} d w d \eta d \eta_{*} .
\end{gathered}
$$

The integrand in (38) coincides with the integrand in the right-hand side of (29), multiplied by the factor $1-\Psi\left(w^{\prime} \geq 0\right) \Psi\left(w_{*}^{\prime} \geq 0\right)$. Using (10) shows that we have to integrate the probability density functions on a bounded domain, which gives a better behavior as $\gamma, \sigma \rightarrow 0$. In more details, while

$$
\begin{gathered}
\int_{\mathbb{R}} \eta^{2} \mu(\eta) d \eta=\sigma^{2}, \\
\int_{\{|\eta|>1-\gamma\}} \eta^{2} \mu(\eta) d \eta \leq \frac{1}{(1-\gamma)^{\alpha}} \int_{\{|\eta|>1-\gamma\}} \eta^{2+\alpha} \mu(\eta) d \eta \leq \sigma^{2+\alpha} E\left(Y^{2+\alpha}\right) .
\end{gathered}
$$

Thus, we can use the same expansion following formula (29) to conclude that $R_{1}(\gamma, \sigma)$ converges to zero as $\gamma \rightarrow 0$.

The second remainder we have to take into account comes out from the fact that in general the total wealth $m(t)$ is not constant in time. Thus we have the additional term

$$
R_{2}(\gamma, \sigma)=-\frac{1}{\gamma} \int_{\mathbb{R}^{2}} \mu(\eta) \mu\left(\eta_{*}\right)\left(\int_{\mathbb{R}_{+}} w_{*} g\left(w_{*}, \tau\right) d w_{*}-m\right) g(w, \tau) \phi^{\prime}(w) d w_{*} d w d \eta d \eta_{*} .
$$

On the other hand, thanks to (29)

$$
\left|\int_{\mathbb{R}_{+}} w_{*} g\left(w_{*}, \tau\right) d w_{*}-m\right| \leq\left[\exp \left\{\frac{\sigma^{2+\alpha}(2-\gamma)}{(1-\gamma)^{2+\alpha}} \tau\right\}-1\right] m
$$

which implies the convergence to zero of the remainder as $\gamma \rightarrow 0$. Hence we proved

Theorem 4.1 Let the probability density $f_{0} \in \mathcal{M}_{p}$, where $p=2+\delta$ for some $\delta>0$, and let the symmetric random variable $Y$ which characterizes the kernel have a density in $\mathcal{M}_{2+\alpha}$, with $\alpha>\delta$. Then, as $\gamma \rightarrow 0, \sigma \rightarrow 0$ in such a way that $\sigma^{2}=\lambda \gamma$ the weak 
solution to the Boltzmann equation for the scaled density $g_{\gamma}(v, \tau)=f(v, t)$, with $\tau=\gamma t$ converges, up to extraction of a subsequence, to a probability density $g(w, \tau)$. This density is a weak solution of the Fokker-Planck equation (30), and it is such that the mean wealth is conserved.

The stationary state of the Fokker-Planck equation can be directly computed and, by assuming for simplicity

$$
m=\int_{\mathbb{R}_{+}} f(w, t) d w=1,
$$

it can be written as

$$
g_{\infty}(w)=\frac{(\mu-1)^{\mu}}{\Gamma(\mu)} \frac{\exp \left(-\frac{\mu-1}{w}\right)}{w^{1+\mu}}
$$

where

$$
\mu=1+\frac{2}{\lambda}>1
$$

Therefore the stationary distribution exhibits a Pareto power law tail for large $w$ 's.

Note that this equation is essentially the same Fokker-Planck equation derived from a Lotka-Volterra interaction in [4, 20, 21].

\section{Numerical results}

In this section, we shall present some numerical test and we shall compare the stationary results obtained by using Monte Carlo simulation of the kinetic model with the stationary state of the Fokker-Planck model. We start from a situation where all individuals shares the same money. At each iteration, we choose randomly two individual. Then, the parameter $\eta$ is choosen accordingly to a normal centered law (one for each individual) and the trade is performed if it is admissible i.e. when the new money of each individual remain positive. We report the results for the density $\tilde{f}(w, t)=m(t) f(m(t) w, t))$ where $m(t)$ is the (variable in time) total amount of money in the system. Note that the density $\tilde{f}$ is normalized to have the first moment equal to one.

We use $N=2000$ individuals and perform several iterations until a stationary state is reached. The distribution is then averaged over the next 250 iterations.

We plot the distribution function for different values of $\left(\gamma, \sigma^{2}\right)=\{(0.1,0.2),(0.01,0.02)\}$.

The values for $\gamma$ and $\sigma^{2}$ are such that we have a fixed $\lambda=2$ corresponding to a coefficient $\mu=2$ which is a realistic value for the income distribution of capitalistic economies [4, 21]. As prescribed from our theoretical analysis we observe that the equilibrium distribution converges toward the function $f_{\infty}$ as both $\gamma$ and $\sigma^{2}$ go to 0 , with $\sigma^{2} / \gamma=2$ (see Figures 1 and 2).

The corresponding behaviors of the total amount of money obtained with $N=10000$ agents are given in Figure 3. Note that, as expected, asymptotically the exponential growth vanishes and the model preserves the total amount of money. 

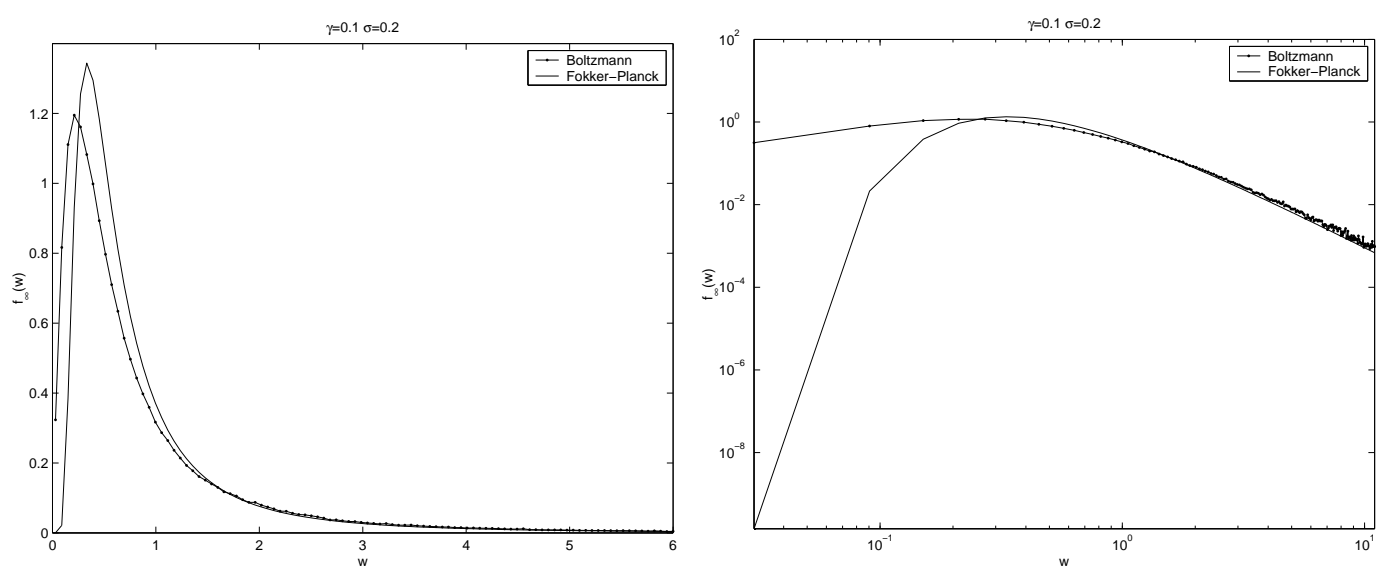

Figure 1: Asymptotic behavior of the Fokker-Planck model and the Boltzmann model for $\mu=2.0, \gamma=0.1$ and $\sigma=0.2$. Figure on the right is in loglog-scale.

\section{Conclusions}

We introduced and discussed a nonlinear kinetic model for a simple market economy, which is based on binary exchanges of money and speculative trading. We showed that at suitably large times, in presence of a large number of trades in which agents exchange a small amount of money, the nonlinear kinetic equation is well-approximated by a linear Fokker-Planck type equation, which admits a stationary steady state with Pareto tails. Convergence towards a similar steady state is shown numerically for the solution of the kinetic model after a suitable normalization which guarantees the conservation of the mean wealth. Our analysis enlightens both the range of validity of the Fokker-Planck equation (36), and why in the continuous trading limit the mean wealth, which is increasing for the kinetic model, remains constant. The present derivation takes advantages of the deep similarities between the trade rule (1) and a molecular dissipative collision. However, it is expected that the asymptotic analysis discussed in this paper is not restricted to the trade rule (1), so that different microscopic interactions could be treated as well. It is clear that the analogy between the trade rule (11) and a one-dimensional molecular dissipative collision suggests in a natural way the continuous trading asymptotic which is well-understood in kinetic theory [26] with the name of quasi-elastic asymptotic procedure. Furthermore, the formation of overpopulated energy tails for large times in the kinetic model is in accord with the analogous result valid for the Boltzmann equation for a dissipative granular Maxwellian gas [1, 9, 10]. Pushing further these analogies may help to clarify many aspects of large-time behavior of market economies.

Acknowledgment: The authors acknowledge support from the IHP network HYKE "Hyperbolic and Kinetic Equations: Asymptotics, Numerics, Applications" HPRN-CT-200200282 funded by the EC., and from the Italian MIUR, project "Mathematical Problems of Kinetic Theories". L. Pareschi thanks the Department of Mathematics and Applications, 

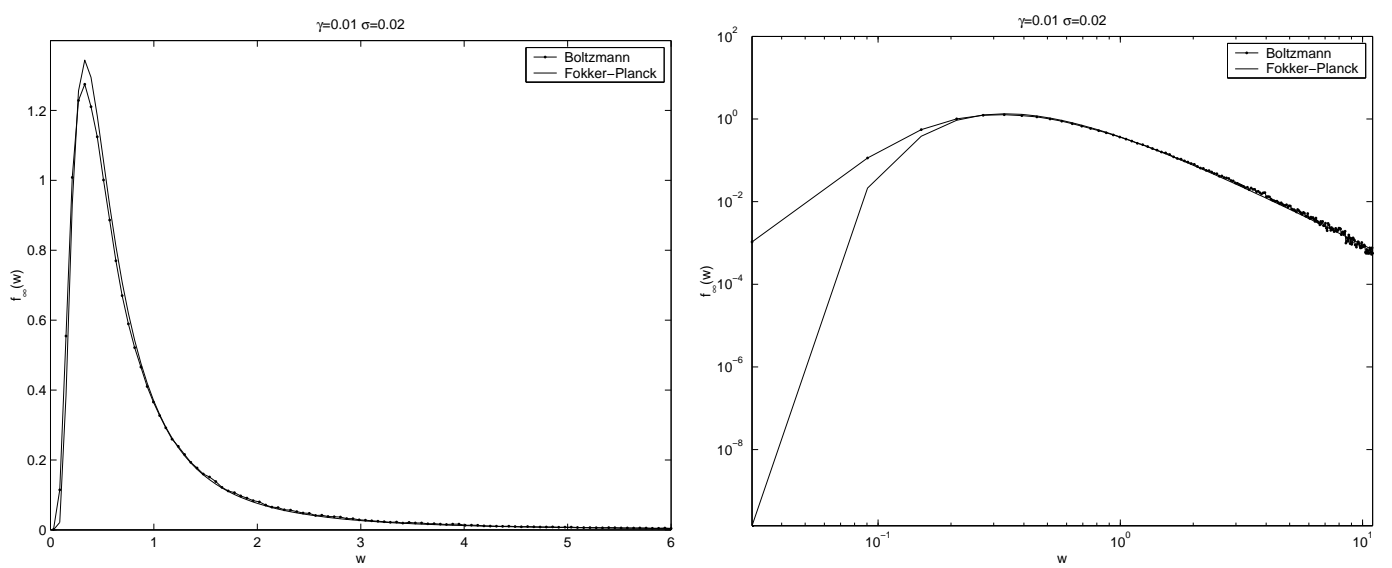

Figure 2: Asymptotic behavior of the Fokker-Planck model and the Boltzmann model for $\mu=2.0, \gamma=0.01$ and $\sigma=0.02$. Figure on the right is in loglog-scale.

Mathematical Physics of Orleans (MAPMO) for the kind hospitality during his visit.

\section{References}

[1] A. Baldassarri. U. Marini Bettolo Marconi, A. Puglisi, Kinetic models of inelastic gases. Mat. Mod. Meth. Appl. Sci. 12 965-983 (2002).

[2] A.V. Bobylev, The theory of the spatially Uniform Boltzmann equation for Maxwell molecules Sov. Sci. Review C 7, 112-229 (1988).

[3] A.V. Bobylev, J.A-Carrillo, I. Gamba, On some properties of kinetic and hydrodynamics equations for inelastic interactions J. Statist. Phys. 98 743-773 (2000).

[4] J.P.Bouchaud, M.Mézard, Wealth condensation in a simple model of economy, Physica A, 282, 536-545 (2000).

[5] C. Cercignani, R. Illner, M. Pulvirenti, The mathematical theory of dilute gases, Springer Series in Applied Mathematical Sciences, Vol. 106 Springer-Verlag 1994.

[6] A.Chakraborti, Distributions of money in models of market economy, Int. J. Modern Phys. C, 13 1315-1321 (2002).

[7] A.Chakraborti, B.K.Chakrabarti, Statistical mechanics of money: how saving propensity affects its distributions, Eur. Phys. J. B., 17, 167-170 (2000).

[8] P.Degond, L.Pareschi, G.Russo, Modelling and numerical methods for kinetic equations, Birkhauser, (2004).

[9] M.H. Ernst, R. Brito, High energy tails for inelastic Maxwell models. Europhys. Lett, 43 497-502 (2002). 



Figure 3: Growth of the total amount of money in the market corresponding to the data of the previous figure.

[10] M.H. Ernst, R. Brito, Scaling solutions of inelastic Boltzmann equation with overpopulated high energy tails. J. Statist. Phys., 109 407-432 (2002).

[11] A.Drăgulescu, V.M.Yakovenko, Statistical mechanics of money, Eur. Phys. J. B., 17, 723-729 (2000).

[12] X. Gabaix, P. Gopikrishnan, V. Plerou, and H. E. Stanley, A Theory of Power-Law Distributions in Financial Market Fluctuations, Nature 423, 267-270 (2003).

[13] B.Hayes, Follow the money, American Scientist, 90 (5), 400-405 (2002).

[14] S.Ispolatov, P.L.Krapivsky, S.Redner, Wealth distributions in asset exchange models, Eur. Phys. J. B, 2, pp.267-276, (1998).

[15] A.Klar, R. Wegener, A Kinetic Model for Vehicular Traffic Derived from a Stochastic Microscopic Model, Transp. Theory Stat. Phys., 25 (7), 785-798 (1996).

[16] H.Levy, M.Levy, S.Solomon, Microscopic simulations of financial markets, Academic Press, New York, (2000).

[17] B.Mandelbrot, The Pareto-Lvy law and the distribution of income, International Economic Review ,1, 79-106, (1960).

[18] R.N.Mantegna, H.E.Stanley, An introduction to econophysics, Cambridge University Press, Cambridge, 2000.

[19] S. McNamara, W.R. Young, Kinetics of a one-dimensional granular medium in the quasi-elastic limit, Phys. Fluids A 5 34-45 (1993). 
[20] S.Solomon, Stochastic Lotka-Volterra systems of competing auto-catalytic agents lead generically to truncated Pareto power wealth distribution, truncated Levy distribution of market returns, clustered volatility, booms and crashes, Computational Finance 97, eds. A-P. N. Refenes, A.N. Burgess, J.E. Moody (Kluwer Academic Publishers 1998).

[21] O.Malcai, O.Biham, S.Solomon, P.Richmond, Theoretical analysis and simulations of the generalized Lotka-Volterra model, Phys. Rev. E, 66, 031102 (2002) (6 pages).

[22] L. Pareschi, Microscopic dynamics and mesoscopic modelling in economy: kinetic equations and numerical methods, in preparation, 2004.

[23] V.Pareto, Cours d'Economie Politique, Lausanne and Paris, 1897.

[24] V. Plerou, P. Gopikrishnan, and H. E. Stanley, Two-Phase Behaviour of Financial Markets, Nature 421, 130, (2003). cond-mat/0111349.

[25] F.Slanina, Inelastically scattering particles and wealth distribution in an open economy, preprint (2003) cond-mat/0311025.

[26] G. Toscani, One-dimensional kinetic models of granular flows, RAIRO Modél Math. Anal. Numér. 34 1277-1292 (2000). 